\title{
Estudo comparativo entre valores teóricos e resultados experimentais de módulo de elasticidade de concretos produzidos com diferentes tipos de agregado graúdo
}

\author{
Comparative study between theoretical values and \\ experimental results of concrete elasticity modulus \\ produced with different types of coarse aggregate
}

\section{Antonio Carlos dos Santos \\ Angela Maria de Arruda \\ Turibio José da Silva \\ Paula de Carvalho Palma Vitor}

Antonio Carlos dos Santos Universidade Federal de Uberlândia Uberlândia - MG - Brasil

Angela Maria de Arruda Universidade Federal de Uberlândia Uberlândia - MG - Brasil

Turibio José da Silva Universidade Federal de Uberlândia Uberlândia - MG - Brasil

Paula de Carvalho Palma Vitor Universidade Federal de Uberlândia Uberlândia - MG - Brasil

Recebido em 05/08/15 Aceito em 25/01/17

\section{Resumo}

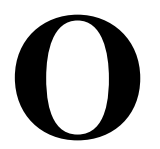

módulo de elasticidade do concreto é uma propriedade importante para os profissionais envolvidos na indústria da construção civil, uma vez que seu valor é determinante para o controle das deformações. Este trabalho avaliou o módulo de elasticidade de três classes distintas de concreto $(\mathrm{C} 20, \mathrm{C} 30$ e $\mathrm{C} 40)$ produzidas com dois tipos litológicos de rochas, basalto e dolomito, de diferentes jazidas da região do Triângulo Mineiro. Como parte do estudo experimental, foram moldadas 324 amostras cilíndricas de $10 \mathrm{~cm} \times$ $20 \mathrm{~cm}$. Os valores de módulo de elasticidade obtidos foram comparados com seis formulações propostas em quatro normas, institutos e códigos do concreto: ABNT NBR 6118 versão 2007 e 2014, ACI 318, EUROCODE 2 and FIB Model Code, Ibracon 2003. Dentre as formulações propostas pelas normas, as indicadas pela FIB Model Code (2010) e ABNT NBR 6118 (2014) apresentaram valores mais próximos aos resultados experimentais deste estudo.

Palavras-chave: Módulo de elasticidade. Agregado graúdo. Concreto.

\begin{abstract}
The elastic modulus is an important property for professionals in the construction industry, since its value is crucial for deformation control. This study evaluates the elastic modulus of three different classes of concrete (C20, C30 and C40), produced with two lithologic types of rocks, basalt and dolomite, from the Triângulo Mineiro region, Brazil. In the experimental study, 324 cylindrical test specimens were cast. The experimental results of the elastic modulus were compared to six formulations proposed in four concrete norms and codes: $A B N T$ NBR 6118, version 2007 and 2014, ACI 318, EUROCODE 2, FIB Model Code and IBRACON (2003). The elastic modulus values obtained with the formulation proposed by FIB Model Code (2010) and ABNT NBR 6118 (2014) were closest to the experimental results of this study.

Keywords: Elastic modulus. Coarse aggregate. Concrete.
\end{abstract}

SANTOS, A. C. dos; ARRUDA, A. M. de; SILVA, T. J. da; VITOR, P. de C. P. Estudo comparativo entre valores teóricos e 281 resultados experimentais de módulo de elasticidade de concretos produzidos com diferentes tipos de agregado graúdo. Ambiente Construído, Porto Alegre, v. 17, n. 3, p. 249-262, jul./set. 2017. ISSN 1678-8621 Associação Nacional de Tecnologia do Ambiente Construído. http://dx.doi.org/10.1590/s1678-86212017000300176 


\section{Introdução}

O módulo de elasticidade do concreto $\left(\mathrm{E}_{\mathrm{c}}\right)$ está associado às deformações estruturais que em excesso podem causar fissuras em alvenarias e gerar outras manifestações patológicas. A estimativa do módulo de elasticidade dos materiais de uma estrutura é importante, pois afeta etapas que influenciam sua vida útil (PEDROSO, 2007), haja vista sua influência na fissuração do concreto.

Os fatores que influenciam o $\mathrm{E}_{\mathrm{c}}$ dependem das características da matriz da pasta de cimento e da zona de transição. A porosidade da matriz afeta a resistência individual da pasta de cimento causando variações no módulo de elasticidade (MELO NETO; HELENE, 2002). Mehta e Monteiro (2014) citam que em idades mais avançadas o $\mathrm{E}_{\mathrm{c}}$ aumenta a uma taxa mais alta do que a resistência à compressão $\left(f_{c}\right)$ em virtude da melhora da densidade da zona de transição na interface. Esses autores também consideram o agregado e os parâmetros de ensaios como fatores que interferem no $\mathrm{E}_{\mathrm{c}}$. Outros fatores influentes sobre os resultados de $E_{c}$ são aqueles inerentes ao processo de ensaio (aplicação da carga, dispositivo de medição, etc.).

Estudos realizados em diversas regiões do Brasil (NUNES, 2005; PACHECO, 2006; ALHADAS, 2008; BARBOSA, 2009) constataram a grande influência da composição mineralógica do agregado sobre o $E_{c}$. Dependendo do tipo do agregado utilizado e da composição do concreto os valores do $\mathrm{E}_{\mathrm{c}}$ podem ter uma variação de até $30 \%$ (MASSUCATO; BASSANI; PAULON, 2003). Fora do Brasil Yang et al. (1995), Zhou, Lydon e Barr (1995), Beshr, Almusallam e Maslehuddin (2003) e Marriaga e Yépez (2011) também verificaram a dependência do módulo de elasticidade da composição mineralógica do agregado.

Segundo Mehta e Monteiro (2014) corpos de prova de concreto que são ensaiados em condições úmidas apresentam $E_{c} 15 \%$ mais alto do que corpos de prova ensaiados em condição seca, e esse resultado independe das dosagens ou do tempo de cura. A secagem surte efeitos opostos para o $E_{c} e f_{c}$. Enquanto o primeiro é reduzido pela ocorrência de microfissurações na zona de transição, a resistência aumenta como consequência das forças de atração nos produtos de hidratação (NEVILLE, 1997). Além da umidade, as condições de carregamento também influenciam o módulo de elasticidade. Segundo Montija (2007) a velocidade de aplicação da carga deve ser uniforme para garantir a uniformidade dos resultados no $\mathrm{E}_{\mathrm{c}}$, já que o concreto não é um material elástico. Quanto maior a velocidade de aplicação da carga, menor a capacidade de propagação das fissuras e da acomodação microestrutural das deformações.

Na literatura são apresentadas diversas formulações com o objetivo de estimar o módulo de elasticidade em função da resistência à compressão do concreto. Na Tabela 1 são citadas as Equações 1 a 5 propostas pela NBR 6118 (ABNT, 2007), NBR 6118 (2014), FIB Model Code (FEDERATION..., 2010), ACI 318 (AMERICAN..., 2011) e EUROCODE 2 (2004), respectivamente.

Onde:

$\mathrm{E}_{\mathrm{ci}}$ é o módulo de elasticidade em GPa;

$\mathrm{f}_{\mathrm{ck}}$ é a resistência característica do concreto à compressão em MPa;

$\alpha$ é um fator que depende do tipo de agregado, sendo 0,9 para calcário, 1,0 para quartzito, gnaisse e granito e 1,2 para basalto;

$\mathrm{E}_{\mathrm{c}}$ é o módulo secante definido como a inclinação da reta que passa pela origem do diagrama $\sigma-\varepsilon$ e pelo ponto correspondente a uma tensão a $0,45 \mathrm{f}_{\mathrm{ck}}$;

$\mathrm{f}_{\mathrm{cm}}$ é a resistência média em $\mathrm{MPa}$; e

$\mathrm{E}_{\mathrm{cs}}$ é o módulo de deformação secante.

Tabela 1 - Equações para estimar o módulo de elasticidade em função da resistência à compressão do concreto apresentadas por várias normativas

\begin{tabular}{l|lc}
\hline \multicolumn{1}{c|}{ Norma } & \multicolumn{1}{c}{ Equação } \\
\hline NBR 6118 (ABNT, 2007) & $E_{c i}=5600 \times f_{c k}^{1 / 2}$ & Eq. 1 \\
\hline NBR 6118 (ABNT, 2014) & $E_{c i}=\alpha_{E} \times 5600 \times f_{c k}^{1 / 2}$ & Eq. 2 \\
\hline FIB Model Code (FEDERATION..., 2010) & $E_{c i}=21500 \times \alpha \times\left(\frac{f_{c m}}{10}\right)^{\frac{1}{3}}$ & Eq. 3 \\
\hline ACI 318 (AMERICAN..., 2011) & $E_{c}=4700 \times f_{c k}^{\frac{1}{2}}$ & Eq. 4 \\
\hline EUROCODE 2 (EUROPEAN..., 2004) & $E_{c s}=22000 \times \alpha_{e} \times\left(\frac{f_{c m}}{10}\right)^{\frac{1}{3}}$ & Eq. 5 \\
\hline
\end{tabular}


Considerando as Equações 4 e 5 propostas respectivamente pelo ACI 318 (AMERICAN..., 2011) e EUROCODE 2 (2004), é possível obter as Equações 6 e 7 correspondentes para $\mathrm{E}_{\mathrm{ci}}$ a partir da Equação 8, proposta pela NBR 6118 (ABNT, 2014).

$E_{c i}=5529,41 \times\left(f_{c k}\right)^{\frac{1}{2}}$

Eq. 6

$E_{c i}=25882,35 \times \alpha_{E} \times\left(\frac{f_{c m}}{10}\right)^{1 / 3}$

$E_{c S}=0,85 \times E_{c i}$

Eq. 8

O Instituto Brasileiro de Concreto (Ibracon) em 2003 sugeriu a Equação 9 como correção da Equação 1 proposta pela NBR 6118 (ABNT, 2007), visando incorporar a esta fórmula dados referentes à consistência do concreto no estado fresco e a influência dos diversos tipos de agregado.

$E_{c i}=a_{1} \times a_{2} \times 5600 f_{c k}^{1 / 2}$

Eq. 9

Onde:

$a_{1}$ é o índice de correção em função da natureza do agregado (1,1 ou 1,2 para basalto denso e calcário sedimentar denso, 1,0 para granito e gnaisse, 0,9 para calcário metamórfico e metassedimento, e 0,7 para arenito); e

$a_{2}$ é determinado em função da consistência do concreto.

A estimativa do $\mathrm{E}_{\mathrm{ci}}$ para idades diferentes de 28 dias pode ser obtida pela Equação 10, proposta pelo FIB Model Code (FEDERATION..., 2010), a qual não estipula um limite de idade do concreto.

Sendo:

$E_{c j}$ o módulo de elasticidade do concreto na idade de $\mathrm{j}$ dias;

$E_{c 28}$ módulo de elasticidade do concreto na idade de 28 dias;

$\mathrm{t}$ a idade do concreto em dias;

s o coeficiente de crescimento da resistência em função do tipo de cimento, 0,2 para CP V ARI; e

0,25 para CP I e CP II e 0,38 para cimento CP III e CP IV.

$E_{c j}=\left\{\exp \left[s \times\left(1-\sqrt{\frac{28}{t}}\right)\right]\right\}^{1 / 2} \times E_{c 28} \quad$ Eq. 10

A NBR 6118 (ABNT, 2014) permite que o $E_{\text {ci }}$ para idades inferiores a 28 dias possa ser estimado pela Equação 11.

Sendo:

$E_{c i}(\mathrm{t})$ o módulo de elasticidade do concreto na idade de t dias;
$E_{c i}$ o módulo de elasticidade do concreto na idade de 28 dias;

$f_{c}(t)$ a resistência à compressão do concreto na idade que se pretende se estimar o $\mathrm{E}_{\mathrm{c}}$, em $\mathrm{MPa}$; e

$f_{c}$ a resistência do concreto à compressão aos 28 dias.

$E_{c i}(t)=\left[\frac{f_{c}(t)}{f_{c}}\right]^{1 / 2} \times E_{c i}$

Eq. 11

Os modelos empíricos propostos pelas normas não são capazes de determinar com exatidão o $\mathrm{E}_{\mathrm{ci}} \mathrm{em}$ função da resistência e do tipo de agregado, fornecendo aproximações dos valores reais (MELO NETO; HELENE, 2002). Existem tentativas de abranger outros parâmetros com fatores de correção vinculados à natureza do agregado graúdo e à consistência do concreto fresco (HELENE; ANDRADE, 2010).

Desse modo, o presente trabalho teve como objetivo comparar os valores experimentais obtidos com os valores estimados de $\mathrm{E}_{\mathrm{ci}}$ pelas equações propostas pelas normas NBR 6118 (ABNT, 2007), NBR 6118 (ABNT, 2014), FIB Model Code (FEDERATION..., 2010), ACI 318 (AMERICAN..., 2011) e EUROCODE 2 (2004), a fim de constatar qual formulação melhor representa o módulo de elasticidade de concretos produzidos com agregados da região do Triângulo Mineiro, MG, Brasil

\section{Materiais e métodos}

Neste estudo foram definidos três valores de $\mathrm{f}_{\mathrm{ck}}, 20$ $\mathrm{MPa}, 30 \mathrm{MPa}$ e $40 \mathrm{MPa}$, e dois tipos de agregado, basalto e dolomito, extraídos de jazidas localizadas em três diferentes cidades, Uberlândia, Uberaba e Patos de Minas, localizadas na região do Triângulo Mineiro no estado de Minas Gerais.

A nomenclatura adotada para as misturas foi composta pela $\mathrm{f}_{\mathrm{ck}}$ do concreto $(\mathrm{C} 20, \mathrm{C} 30, \mathrm{C} 40)$, tipo de agregado graúdo utilizado (BA-basalto; DOdolomito) e jazidas onde foram extraídos tais agregados:

(a) UDIA, jazida localizada na cidade de Uberlândia;

(b) PA, jazida localizada na cidade de Patos de Minas; e

(c) UBERA, jazida localizada na cidade de Uberaba.

Por exemplo, o corpo de prova C20-BA-UDIA se refere ao CP moldado com concreto classe $20 \mathrm{MPa}$, agregado graúdo basalto retirado da jazida da cidade de Uberlândia. A Figura 1 ilustra o material que constituiu os agregados. 
Figura 1 - Amostras das rochas

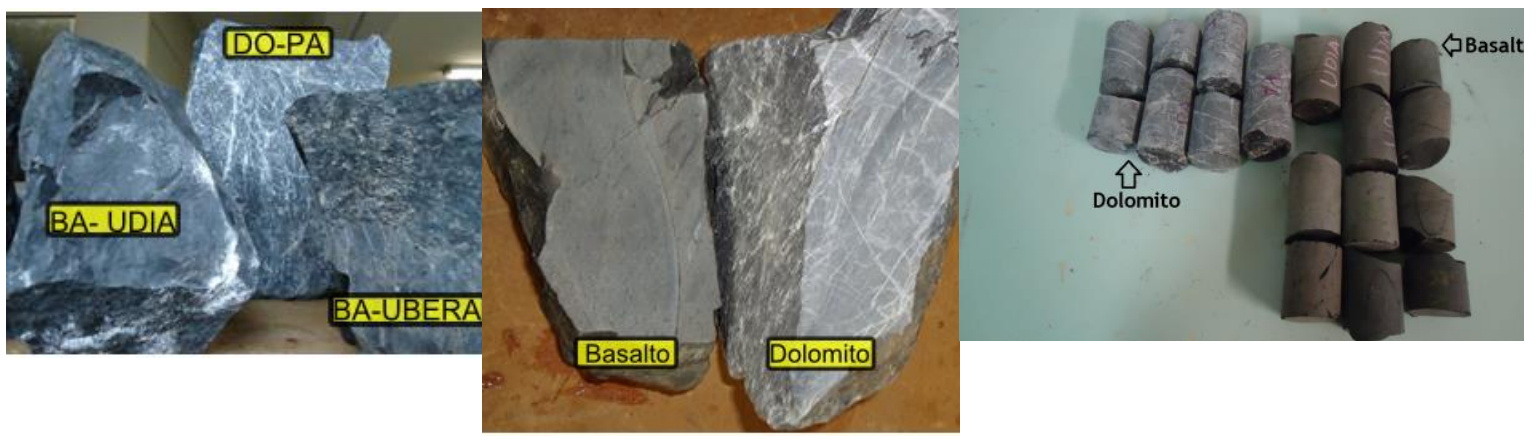

Conforme fluxograma da Figura 2, para cada traço de concreto foram moldados 36 CPs cilíndricos (10 $\mathrm{cm} \times 20 \mathrm{~cm}$ ). Nas idades de 7 dias, 14 dias, 28 dias e 56 dias foram realizados os ensaios para determinação da resistência à compressão e módulo de elasticidade.

Os ensaios do concreto endurecido foram realizados no laboratório de materiais de construção civil da Universidade Federal de Uberlândia, Brasil, utilizando a prensa eletromecânica EMIC ${ }^{\circledR}$ modelo DL-60000, servo-controlada. A prensa possui capacidade de carga de 600 kN. A mensuração da deformação ocorreu através de dois canais de extensômetros.

O diâmetro dos CPs foi determinado com exatidão de $\pm 0.1 \mathrm{~mm}$, pela média de dois diâmetros medidos ortogonalmente na metade da altura do CP. A altura dos CPs também foi determinada com $\pm 0.1 \mathrm{~mm}$ de precisão. As faces de aplicação de carga dos CPs (base e topo) foram regularizadas com enxofre.

$\mathrm{O}$ ensaio de resistência à compressão foi realizado com carga aplicada continuamente e sem choques, com velocidade de carregamento constante de $(0,45$ $\pm 0,15) \mathrm{MPa} / \mathrm{s}$.

Para a realização do ensaio de determinação do $\mathrm{E}_{\mathrm{ci}}$ foram utilizados extensômetros elétricos de ancoragem tipo clip gage (ver Figura 3).

$\mathrm{O}$ ensaio para obtenção do $\mathrm{E}_{\mathrm{ci}}$ foi realizado conforme a norma brasileira NBR 8522 (ABNT, 2008).

Para a moldagem dos corpos de prova, o concreto foi dosado com cimento CP-II-E-32 de resistência à compressão média aos 28 dias de 31,27 $\mathrm{MPa}$. Utilizou-se como agregado miúdo a areia comum de leito de rio com módulo de finura de 2,53. Foram utilizados agregados graúdos de diâmetro máximo de $19 \mathrm{~mm}$ selecionados de dois tipos de rocha, basalto (BA) e dolomito (DO), extraídos de jazidas de três cidades diferentes da região do Triângulo
Mineiro, estado de Minas Gerais, Brasil. Na Tabela 2 podem ser visualizadas as características físicas de cada tipo de agregado graúdo. Para a obtenção das propriedades das rochas foram extraídos testemunhos $(5 \mathrm{~cm} \times 10 \mathrm{~cm})$ para ensaio de resistência à compressão e módulo de elasticidade. Entretanto, as amostras apresentaram fissuras facilmente perceptíveis. Verificou-se também que algumas das rochas apresentavam fissuras em vários blocos analisados no próprio local da retirada. Durante a extração, a maioria dos testemunhos não atingiu a altura esperada $(10 \mathrm{~cm})$, visto que a presença de fissuras internas nas amostras favoreceu a ruptura nesses pontos. Devido aos problemas encontrados nos testemunhos, a caracterização da rocha não foi efetuada, tomandose para efeito deste trabalho.

$\mathrm{O}$ aditivo e a água utilizados na dosagem do concreto foram o superplastificante com base química de policarboxilato e água potável proveniente da rede de distribuição local, respectivamente.

O modelo de dosagem utilizado foi o método IPT/EPUSP (Instituto de Pesquisa Tecnológicas do Estado de São Paulo) (1992). Os valores de relação água/cimento $(\mathrm{a} / \mathrm{c}) \quad 0,53,0,45$ e 0,35 foram determinados de modo que fossem atingidos os valores dos $\mathrm{f}_{\mathrm{ck}}$ propostos. O consumo dos materiais para um metro cúbico de concreto e os traços em massa estão apresentados na Tabela 3.

Após a determinação do abatimento de tronco de cone conforme NBR NM 67 (ABNT, 1998) e massa específica, o material foi inserido na betoneira e misturado por cerca de 2 minutos, para então conduzir a moldagem dos CPs. Vale ressaltar que o abatimento de tronco de cone não foi fixado, visto que a variável de controle foi a resistência à compressão previamente definida, sendo necessário manter a relação água/cimento da dosagem. 
Figura 2 - Fluxograma de moldagem dos corpos de prova

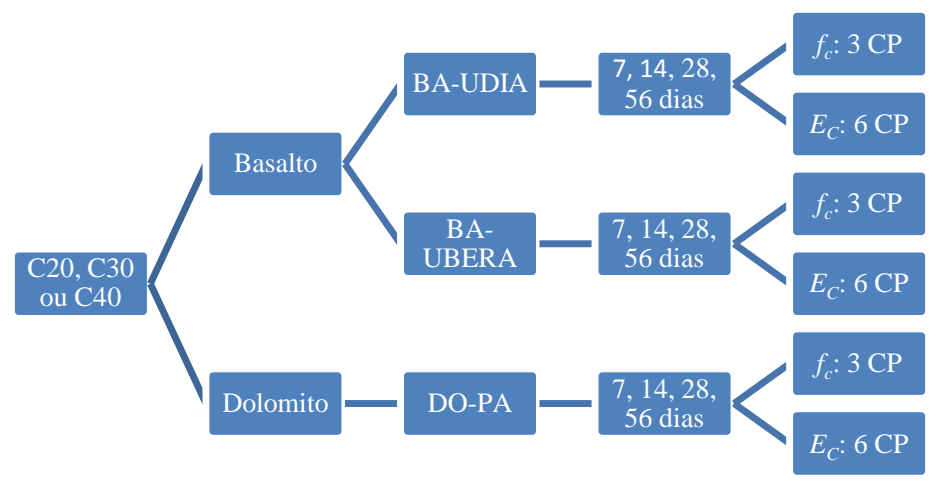

Figura 3 - Clip gages posicionados no corpo de prova para o ensaio de determinação do $E_{c i}$

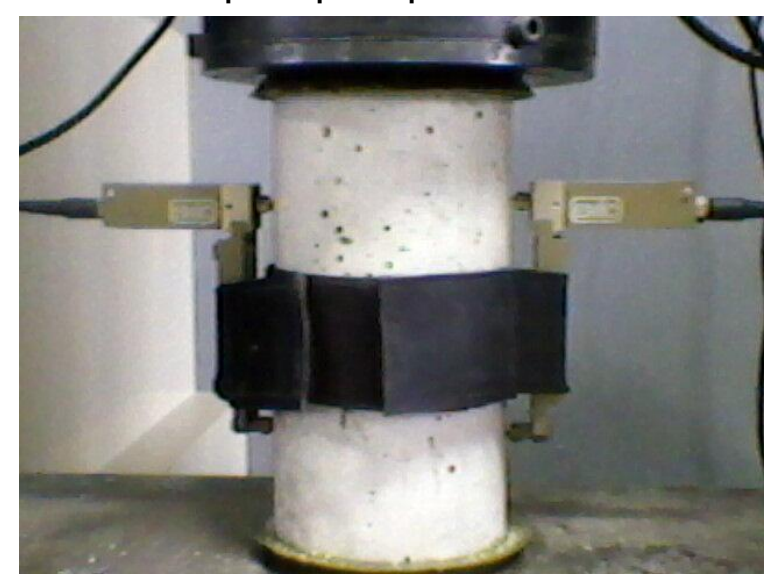

Tabela 2 - Características físicas dos agregados graúdos

\begin{tabular}{l|c|c|c}
\hline \multicolumn{1}{c|}{ Propriedades } & BA-UDIA & BA-UBERA & DO-PA \\
\hline Massa específica $\left(\mathrm{g} / \mathrm{cm}^{3}\right)$ & 2,84 & 2,90 & 2,69 \\
Massa unitária $\left(\mathrm{g} / \mathrm{cm}^{3}\right)$ & 1,53 & 1,53 & 1,42 \\
Dimensão máxima $(\mathrm{mm})$ & 19 & 19 & 19 \\
Módulo de finura & 6,98 & 7,73 & 6,96 \\
Teor de materiais pulverulentos $(\%)$ & 0,77 & 0,52 & 0,33 \\
\hline
\end{tabular}

Tabela 3 - Traços em massa e consumo dos materiais

\begin{tabular}{|c|c|c|c|c|c|c|c|c|c|}
\hline \multirow[b]{2}{*}{ Materiais } & \multicolumn{3}{|c|}{$\mathrm{C20}$} & \multicolumn{3}{|c|}{$\mathrm{C} 30$} & \multicolumn{3}{|c|}{$\mathrm{C40}$} \\
\hline & $\begin{array}{l}\text { BA- } \\
\text { UDIA }\end{array}$ & DO-PA & $\begin{array}{c}\text { BA- } \\
\text { UBERA }\end{array}$ & $\begin{array}{l}\text { BA- } \\
\text { UDIA }\end{array}$ & DO-PA & $\begin{array}{c}\text { BA- } \\
\text { UBERA }\end{array}$ & $\begin{array}{l}\text { BA- } \\
\text { UDIA }\end{array}$ & DO-PA & $\begin{array}{c}\text { BA- } \\
\text { UBERA }\end{array}$ \\
\hline $\begin{array}{c}\text { Cimento CP } \\
\text { II-E } \\
32\left(\mathrm{~kg} / \mathrm{m}^{3}\right) \\
\end{array}$ & 329 & 322 & 332 & 389 & 380 & 392 & 470 & 459 & 474 \\
\hline $\begin{array}{c}\text { Areia } \\
\left(\mathrm{kg} / \mathrm{m}^{3}\right)\end{array}$ & 822,50 & 805,00 & 830,00 & 778,00 & 760,00 & 784,00 & 705,00 & 688,50 & 711,00 \\
\hline $\begin{array}{c}\text { Brita } \\
\left(\mathrm{kg} / \mathrm{m}^{3}\right)\end{array}$ & 1151,50 & 1127,00 & 1162,00 & 1167,00 & 1140,00 & 1176,00 & 1175,00 & 1147,50 & 1185,00 \\
\hline Água $\left(1 / m^{3}\right)$ & 174,37 & 170,66 & 175,96 & 167,27 & 163,40 & 168,56 & 164,50 & 160,65 & 165,90 \\
\hline Fator a/c & 0,53 & 0,53 & 0,53 & 0,43 & 0,43 & 0,43 & 0,35 & 0,35 & 0,35 \\
\hline $\begin{array}{c}\text { Traço em } \\
\text { massa }\end{array}$ & \multicolumn{3}{|c|}{$1: 2,5: 3,5: 0,53$} & \multicolumn{3}{|c|}{$1: 2: 3: 0,43$} & \multicolumn{3}{|c|}{$1: 1,5: 2,5: 0,35$} \\
\hline
\end{tabular}

Estudo comparativo entre valores teóricos e resultados experimentais de módulo de elasticidade de concretos 
Para a moldagem dos CPs foram utilizadas fôrmas metálicas cilíndricas de dimensões $10 \mathrm{~cm} \times 20 \mathrm{~cm}$. $\mathrm{O}$ adensamento do concreto foi feito de forma manual, em duas camadas e 12 golpes por camada. Os CPs foram mantidos em câmara úmida até 24 horas após a moldagem, após esse período foram transferidos para um tanque onde permaneceram submersos até a data de realização de cada ensaio.

\section{Resultados e discussão}

\section{Análise da influência do agregado}

Nas Tabelas 4 e 5 são apresentados os resultados médios da resistência à compressão $\left(\mathrm{f}_{\mathrm{cm}}\right)$ e módulo de elasticidade $\left(\mathrm{E}_{\mathrm{cm}}\right)$ com seus respectivos desviospadrão $\left(S_{d}\right)$ e coeficiente de variação $(\mathrm{COV})$. É possível verificar que todos os traços atingiram o $\mathrm{f}_{\mathrm{ck}}$ especificado na dosagem.
Observa-se nas Tabelas 4 e Tabela 5 que os coeficientes de variação obtidos foram, em sua maioria, inferiores a $10 \%$, o que indica uniformidade no processo.

As diferenças na composição mineralógica dos agregados graúdos afetam a resistência do concreto. $\mathrm{O}$ concreto produzido com agregado DO, independentemente da classe de resistência, foi aquele que apresentou menor ganho de $\mathrm{f}_{\mathrm{c}}$ dos 7 aos 56 dias, $11,67 \%$ em média. Para os concretos produzidos com agregado BA, exceto para a classe C40, o concreto BA-UBERA apresentou maior ganho de resistência com o tempo, em média $31,58 \%$. Observa-se que, conforme afirmado por Mehta e Monteiro (2014), a substituição de um agregado de origem sedimentar por um agregado de origem basáltica eleva a resistência na ligação na interface.

Tabela 4 - Abatimento, massa específica, resistência à compressão média $\left(f_{c m}\right)$, desvio-padrão $\left(S_{d}\right)$ e coeficiente de variação (COV) para todos os traços

\begin{tabular}{|c|c|c|c|c|c|c|c|c|c|}
\hline \multicolumn{3}{|c|}{ Traços } & \multirow{3}{*}{$\begin{array}{l}\text { Abatimento } \\
\text { (mm) }\end{array}$} & \multirow{3}{*}{$\begin{array}{c}\text { Massa } \\
\text { específica } \\
\left(\mathrm{g} / \mathrm{cm}^{3}\right)\end{array}$} & \multicolumn{5}{|c|}{ Resistência à compressão axial (MPa) } \\
\hline \multirow{2}{*}{ Classe } & \multirow{2}{*}{$\begin{array}{c}\text { Tipo } \\
\text { agregado }\end{array}$} & \multirow{2}{*}{ Jazida } & & & \multirow{2}{*}{ Variáveis } & \multicolumn{4}{|c|}{ Idade (dias) } \\
\hline & & & & & & 7 & 14 & 28 & 56 \\
\hline \multirow{9}{*}{$\mathrm{C} 20$} & \multirow{6}{*}{$\mathrm{BA}$} & \multirow{3}{*}{ UDIA } & \multirow{3}{*}{100} & \multirow{3}{*}{2,23} & $f_{c m}(\mathrm{MPa})$ & 22,87 & 24,44 & 25,57 & 26,19 \\
\hline & & & & & $\mathrm{S}_{\mathrm{d}}(\mathrm{MPa})$ & 1,63 & 1,59 & 2,24 & 2,12 \\
\hline & & & & & $\mathrm{COV}(\%)$ & 7,13 & 6,51 & 8,76 & 8,09 \\
\hline & & \multirow{3}{*}{ UBERA } & \multirow{3}{*}{150} & \multirow{3}{*}{2,41} & $f_{c m}(\mathrm{MPa})$ & 26,88 & 29,69 & 35,5 & 36,61 \\
\hline & & & & & $\mathrm{S}_{\mathrm{d}}(\mathrm{MPa})$ & 1,67 & 2,33 & 1,36 & 1,89 \\
\hline & & & & & $\operatorname{COV}(\%)$ & 6,21 & 7,85 & 3,83 & 5,16 \\
\hline & \multirow{3}{*}{ DO } & \multirow{3}{*}{ PA } & \multirow{3}{*}{70} & \multirow{3}{*}{2,26} & $f_{c m}(\mathrm{MPa})$ & 25,65 & 28,73 & 29,48 & 29,82 \\
\hline & & & & & $\mathrm{S}_{\mathrm{d}}(\mathrm{MPa})$ & 1,06 & 1,81 & 1,7 & 1,65 \\
\hline & & & & & $\operatorname{COV}(\%)$ & 4,13 & 6,30 & 5,77 & 5,53 \\
\hline \multirow{9}{*}{ C30 } & \multirow{6}{*}{ BA } & \multirow{3}{*}{ UDIA } & \multirow{3}{*}{200} & \multirow{3}{*}{2,28} & $f_{c m}(\mathrm{MPa})$ & 32,22 & 35,67 & 35,78 & 36,29 \\
\hline & & & & & $\mathrm{S}_{\mathrm{d}}(\mathrm{MPa})$ & 1,98 & 2,65 & 3,15 & 2,12 \\
\hline & & & & & $\mathrm{COV}(\%)$ & 6,15 & 7,43 & 8,80 & 5,84 \\
\hline & & \multirow{3}{*}{ UBERA } & \multirow{3}{*}{160} & \multirow{3}{*}{2,46} & $f_{c m}(\mathrm{MPa})$ & 36,26 & 39,33 & 42,18 & 46,04 \\
\hline & & & & & $\mathrm{S}_{\mathrm{d}}(\mathrm{MPa})$ & 1,73 & 1,48 & 0,85 & 3,93 \\
\hline & & & & & $\operatorname{COV}(\%)$ & 4,77 & 3,76 & 2,02 & 8,54 \\
\hline & & & & & $f_{c m}(\mathrm{MPa})$ & 32,1 & 35,63 & 35,61 & 36,72 \\
\hline & DO & PA & 170 & 2,29 & $\mathrm{~S}_{\mathrm{d}}(\mathrm{MPa})$ & 2,44 & 2,11 & 1,08 & 1,39 \\
\hline & & & & & $\operatorname{COV}(\%)$ & 7,60 & 5,92 & 3,03 & 3,79 \\
\hline & & & & & $f_{c m}(\mathrm{MPa})$ & 46,49 & 51,72 & 53,63 & 56 \\
\hline & & UDIA & 100 & 2,50 & $\mathrm{~S}_{\mathrm{d}}(\mathrm{MPa})$ & 5,39 & 4,98 & 3,83 & 5,85 \\
\hline & RA & & & & $\operatorname{COV}(\%)$ & 11,59 & 9,63 & 7,14 & 10,45 \\
\hline & BA & & & & $f_{c m}(\mathrm{MPa})$ & 47,46 & 49,9 & 49,5 & 50,29 \\
\hline $\mathrm{C} 40$ & & UBERA & 150 & 2,55 & $\mathrm{~S}_{\mathrm{d}}(\mathrm{MPa})$ & 3,35 & 2,81 & 2,89 & 2,12 \\
\hline & & & & & $\mathrm{COV}(\%)$ & 7,06 & 5,63 & 5,84 & 4,22 \\
\hline & & & & & $f_{c m}(\mathrm{MPa})$ & 49,86 & 50,44 & 49,54 & 52,04 \\
\hline & DO & PA & 70 & 2,43 & $\mathrm{~S}_{\mathrm{d}}(\mathrm{MPa})$ & 3,87 & 4,47 & 2 & 4,98 \\
\hline & & & & & $\operatorname{COV}(\%)$ & 7,76 & 8,86 & 4,04 & 9,57 \\
\hline
\end{tabular}

Nota: Classe: classe de resistência; Tipo: tipo de agregado; e Jazida: jazida de origem do agregado. 
Tabela 5 - Módulo de elasticidade médio $\left(\mathrm{E}_{\mathrm{cim}}\right)$, desvio-padrão $\left(\mathrm{S}_{\mathrm{d}}\right)$ e coeficiente de variação $(\mathrm{COV})$ para todos os traços

\begin{tabular}{|c|c|c|c|c|c|c|c|}
\hline \multicolumn{3}{|c|}{ Traços } & \multicolumn{5}{|c|}{ Módulo de elasticidade (GPa) } \\
\hline \multirow{2}{*}{ Classe } & \multirow{2}{*}{ Tipo } & \multirow{2}{*}{ Jazida } & \multirow{2}{*}{ Variáveis } & \multicolumn{4}{|c|}{$\begin{array}{l}\text { Idade (dias) } \\
\end{array}$} \\
\hline & & & & 7 & 14 & 28 & 56 \\
\hline \multirow{9}{*}{$\mathrm{C} 20$} & \multirow{6}{*}{ BA } & \multirow{3}{*}{ UDIA } & $E_{\text {cim }}(\mathrm{GPa})$ & 31,3 & 32,4 & 35,06 & 37,77 \\
\hline & & & $\mathrm{S}_{\mathrm{d}}(\mathrm{GPa})$ & 1,58 & 3,64 & 1,91 & 1,06 \\
\hline & & & $\operatorname{COV}(\%)$ & 5,05 & 11,23 & 5,45 & 2,81 \\
\hline & & \multirow{3}{*}{ UBERA } & $E_{\text {cim }}(\mathrm{GPa})$ & 36,5 & 42,32 & 42,38 & 43,02 \\
\hline & & & $\mathrm{S}_{\mathrm{d}}(\mathrm{GPa})$ & 2,88 & 4,39 & 1,25 & 4,24 \\
\hline & & & $\operatorname{COV}(\%)$ & 7,89 & 10,37 & 2,95 & 9,86 \\
\hline & \multirow{3}{*}{ DO } & \multirow{3}{*}{ PA } & $E_{\text {cim }}(\mathrm{GPa})$ & 36,35 & 36,98 & 37,24 & 37,68 \\
\hline & & & $\mathrm{S}_{\mathrm{d}}(\mathrm{GPa})$ & 1,72 & 1,51 & 2,3 & 1,98 \\
\hline & & & $\operatorname{COV}(\%)$ & 4,73 & 4,08 & 6,18 & 5,25 \\
\hline \multirow{9}{*}{ C30 } & \multirow{6}{*}{ BA } & \multirow{3}{*}{ UDIA } & $E_{\text {cim }}(\mathrm{GPa})$ & 38,39 & 39,47 & 40,96 & 43,65 \\
\hline & & & $\mathrm{S}_{\mathrm{d}}(\mathrm{GPa})$ & 2,92 & 1,83 & 3,16 & 1,95 \\
\hline & & & $\operatorname{COV}(\%)$ & 7,61 & 4,64 & 7,71 & 4,47 \\
\hline & & \multirow{3}{*}{ UBERA } & $E_{\text {cim }}(\mathrm{GPa})$ & 40,65 & 42,35 & 43,17 & 46,4 \\
\hline & & & $\mathrm{S}_{\mathrm{d}}(\mathrm{GPa})$ & 3,71 & 4,06 & 5,72 & 4,3 \\
\hline & & & $\mathrm{COV}(\%)$ & 9,13 & 9,59 & 13,25 & 9,27 \\
\hline & \multirow{3}{*}{ DO } & \multirow{3}{*}{ PA } & $E_{\text {cim }}(\mathrm{GPa})$ & 40,08 & 39,9 & 41,5 & 42 \\
\hline & & & $\mathrm{S}_{\mathrm{d}}(\mathrm{GPa})$ & 1,72 & 0,44 & 0,92 & 4,66 \\
\hline & & & $\operatorname{COV}(\%)$ & 4,29 & 1,10 & 2,22 & 11,10 \\
\hline \multirow{9}{*}{$\mathrm{C} 40$} & \multirow{6}{*}{ BA } & \multirow{3}{*}{ UDIA } & $E_{\text {cim }}(\mathrm{GPa})$ & 46,72 & 46,13 & 52,8 & 52,73 \\
\hline & & & $\mathrm{S}_{\mathrm{d}}(\mathrm{GPa})$ & 2,02 & 1,59 & 2,9 & 4,1 \\
\hline & & & COV (\%) & 4,32 & 3,45 & 5,49 & 7,78 \\
\hline & & \multirow{3}{*}{ UBERA } & $E_{\text {cim }}(\mathrm{GPa})$ & 45,26 & 45,72 & 53,7 & 56,72 \\
\hline & & & $\mathrm{S}_{\mathrm{d}}(\mathrm{GPa})$ & 2,01 & 4,74 & 5,07 & 2,14 \\
\hline & & & $\mathrm{COV}(\%)$ & 4,44 & 10,37 & 9,44 & 3,77 \\
\hline & \multirow{3}{*}{ DO } & \multirow{3}{*}{ PA } & $E_{c i m}(\mathrm{GPa})$ & 48,97 & 48,53 & 52,45 & 53,38 \\
\hline & & & $\mathrm{S}_{\mathrm{d}}(\mathrm{GPa})$ & 2,1 & 1,25 & 3,18 & 2,59 \\
\hline & & & $\operatorname{COV}(\%)$ & 4,29 & 2,58 & 6,06 & 4,85 \\
\hline
\end{tabular}

Nota: Classe: classe de resistência; Tipo: tipo de agregado; e Jazida: jazida de origem do agregado.

O concreto produzido com agregado DO também apresentou o menor ganho de $\mathrm{E}_{\mathrm{ci}}$ dos 7 dias aos 56 dias, 5,81\% em média. Segundo Mehta e Monteiro (2014), a porosidade do agregado determina sua rigidez, o que por sua vez controla a capacidade do agregado de restringir a deformação da matriz. Assim, o agregado DO, de origem sedimentar, mais poroso apresentou menor capacidade de restringir as deformações. Para os concretos produzidos com agregado BA, exceto para a classe $\mathrm{C} 20$, o concreto BA-UBERA apresentou maior ganho de $\mathrm{E}_{\mathrm{ci}}$ com o tempo, 19,73\%. Esse fato reforça a tendência de que quanto maior a $f_{c}$, maior a influência do tipo de agregado no $E_{\text {ci. }}$ Neville (1997) explica que com o aumento da relação a/c e consequentemente redução da $f_{c}$ a influência do agregado diminui, pois, a influência da resistência da pasta de cimento hidratada sobrepõe ao tipo de agregado.

É sabido da relação inversa entre porosidade e resistência dos sólidos (MEHTA; MONTEIRO, 2014). Para o concreto C20, a pasta de cimento, menos resistente por ser mais porosa, devido ao maior fator a/c da mistura (NEVILLE, 1997; MEHTA; MONTEIRO, 2014), foi determinante na diferença entre os módulos dos concretos produzidos com agregados Ba-UBERA e BAUDIA e sobrepôs-se ao efeito do agregado.

Melo Neto e Helene (2002) estudaram a influência da relação a/c e no módulo de elasticidade do concreto. Eles comprovaram que a porosidade da matriz influenciada pela relação a/c afeta a resistência individual da pasta de cimento causando variações no módulo de elasticidade.

Os concretos da classe C30 dosados com os agregados BA-UDIA e BA-UBERA apresentaram praticamente o mesmo ganho de $\mathrm{E}_{\mathrm{ci}}$. Para o traço C40 os concretos produzidos com agregado extraído de UBERA apresentaram o dobro de ganho se comparados aos produzidos com agregado de UDIA, ou seja, a matriz menos porosa, resultado de uma relação a/c baixa, propiciou a transferência de esforços para o agregado, cuja densidade contribui para a rigidez do compósito. 
Para verificar um dos objetivos deste trabalho, a influência do agregado graúdo no modulo de $\mathrm{E}_{\mathrm{ci}}$, realizou-se a análise de variância, cujos resultados estão na Tabela 6. Inicialmente analisou-se a influência dos basaltos nos valores de $\mathrm{E}_{\mathrm{ci}}$, tomandose somente os valores aos 28 dias de idade. A segunda análise foi entre os resultados de $\mathrm{E}_{\mathrm{ci}}$, aos 28 dias de idade, para os concretos com BA e com DO. A terceira análise foi realizada com a utilização dos resultados de $\mathrm{E}_{\mathrm{ci}}$, em todas as idades, para os concretos com BA e com DO.

Conforme pode ser verificado na Tabela 6 , não houve evidências sobre a influência significativa dos basaltos nos valores de $\mathrm{E}_{\mathrm{ci}}$, tomando-se somente os valores aos 28 dias de idade, exceto para o C20. Quando comparados os valores de $\mathrm{E}_{\mathrm{ci}}$ aos 28 dias de idade dos concretos de todas as classes, preparados com BA e DO, verificou-se que não houve influência significativa dos agregados. Por último, quanto aos valores de $\mathrm{E}_{\mathrm{ci}}$ para todas as idades dos concretos de todas as classes, preparados com BA e DO, também se verificou que não houve evidências sobre a influência significativa dos agregados, exceto para o C30.

\section{Estimativa do módulo de elasticidade}

Nas Figuras 4a, 4b e 4c encontram-se gráficos comparativos da evolução do módulo de elasticidade obtido pela Equação 10 e pela Equação 11 e obtido experimentalmente para os concretos dosados com agregado graúdo BA-UDIA, DO-PA e BA-UBERA até o limite de idade de 56 dias. O valor de s adotado para o cálculo foi 0,25 . A relação entre as resistências poderá ser obtida pela Equação 12 do FIB Model Code (2010).

$\frac{f_{c}(t)}{f_{c}}=\exp \left[S \times\left(1-\sqrt{\frac{28}{t}}\right)\right]$

Eq. 12

Os valores de $\mathrm{E}_{\mathrm{ci}}$ encontrados para traço C30-BAUDIA se mantiveram acima do estimado pelas Equações 10 e 11 independentemente da idade analisada, enquanto o traço C40-BA-UDIA se manteve abaixo do proposto em todas as idades. $\mathrm{O}$ concreto C20-BA-UDIA, após os 28 dias, teve uma taxa de crescimento do $\mathrm{E}_{\mathrm{ci}}$ superior ao concreto C30-BA-UDIA. Tal fato pode ser explicado pela maior disponibilidade de água presente no primeiro traço que propiciou a contínua hidratação do cimento. Os resultados para os três traços, C20-DOPA, C30-DO-PA e C40-DO-PA, foram superestimados pela Equações 10 e 11 após os 28 dias. Para os concretos produzidos com o agregado graúdo BA-UBERA, as Equações 10 e 11, após os 28 dias, superestimou somente o valor de $\mathrm{E}_{\mathrm{ci}}$ do traço C20-BA-UBERA.

Tabela 6 - Análise de variância

\begin{tabular}{c|c|c|c|c|c|c|c}
\hline Análise entre grupos & SQ & gl & MQ & F & valor-P & F crítico & Influência \\
\hline C20 BASALTO 28 DIAS & 242,101 & 1 & 242,1008 & 12,0256 & 0,0060 & 4,9646 & SIM \\
C30 BASALTO 28 DIAS & 13,441 & 1 & 13,4408 & 0,6595 & 0,4356 & 4,9646 & NÃO \\
C40 BASALTO 28 DIAS & 9,520 & 1 & 9,5202 & 0,5386 & 0,4840 & 5,3177 & NÃO \\
C20 28 DIAS BA x DO & 15,340 & 1 & 15,3403 & 0,5191 & 0,4816 & 4,4940 & NÃO \\
C30 28 DIAS BA x DO & 1,322 & 1 & 1,3225 & 0,0910 & 0,7667 & 4,4940 & NÃO \\
C40 28 DIAS BA x DO & 14,700 & 1 & 14,7000 & 0,9794 & 0,3404 & 4,6672 & NÃO \\
C20 TODAS IDADES BA x DO & 7,887 & 1 & 7,8867 & 0,3331 & 0,5657 & 3,9778 & NÃO \\
C30 TODAS IDADES BA x DO & 56,877 & 1 & 56,8767 & 4,0990 & 0,0467 & 3,9778 & SIM \\
C40 TODAS IDADES BA x DO & 7,490 & 1 & 7,4895 & 0,3078 & 0,5812 & 4,0069 & NÃO \\
\hline
\end{tabular}

Nota: Legenda:

SQ é a soma dos quadrados de todos os desvios em relação à média de todas as observações (entre e dentro das amostras);

gl é o grau de liberdade; e

MQ é a média quadrática (entre e dentro das amostras).

$\mathrm{F}=\frac{\text { variância entre amostras }}{\text { variância dentro das amostras }}$

valor-P é a probabilidade da hipótese nula ser verdadeira. Adotou-se o nível de significância do teste de 0,05. $\mathrm{F}$ crítico é o valor calculado em função do nível de significância e dos graus de liberdade da variância entre e dentro das amostras. 
Figura 4 - Comparação entre o módulo de elasticidade obtido pela Equação 10 e Equação 11 e o módulo de elasticidade obtido experimentalmente para os traços produzidos com agregado graúdo (a) BAUDIA, (b) DO-PA e(c) BA-UBERA
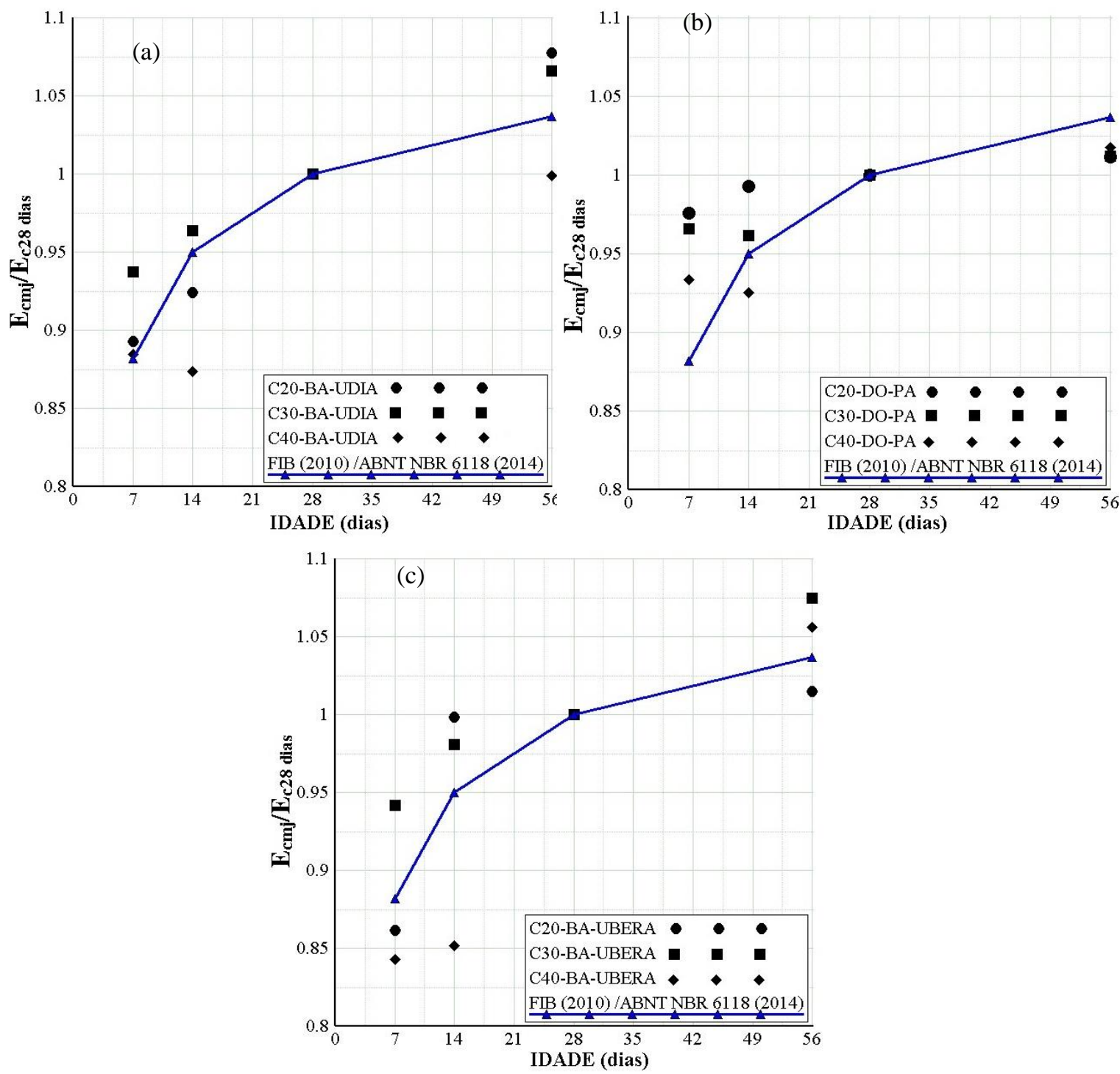

Nos gráficos das Figuras 5a, 5b e 5c apresentam-se os $E_{\text {ci }}$ em função do $f_{c}$ dos concretos estudados para todas as idades e as equações que melhor se ajustam aos resultados encontrados. Foi realizado o ajuste de equação mediante regressão não linear, resultando nas equações indicadas nas Figuras 5a, 5 b e 5c. Para tal foi utilizada a função exponencial. Os coeficientes de determinação obtidos foram 0,83 e 0,81 para os resultados e para a regressão, dos agregados BA-UDIA e DO-PA, ou seja, mais de $80 \%$ das variáveis dependentes conseguem ser explicadas pelo modelo. Entretanto, o concreto preparado com agregado graúdo UBERA apresentou uma dispersão maior que os demais e o coeficiente de determinação do ajuste foi de 0,37 .
$\mathrm{Na}$ seção Introdução foram apresentadas as Equações normativas 1 a 5 que correlacionam $\mathrm{f}_{\mathrm{c}}$ e o módulo de elasticidade. Algumas dessas formulações estimam o módulo de elasticidade a partir da $f_{\text {ck. }}$. Para permitir a comparação, os valores de $\mathrm{f}_{\mathrm{ck}}$ foram obtidos através da Equação 13, sendo $\mathrm{f}_{\mathrm{cm}}$ a resistência à compressão média e $\mathrm{S}_{\mathrm{d}} \mathrm{o}$ desviopadrão encontrado para cada grupo de amostras ensaiado.

$f_{c k}=f_{c m}-1.65 \times S_{d}$

O comparativo dos resultados de $\mathrm{E}_{\mathrm{ci}}$ obtidos experimentalmente nestre trabalho para cada tipo de concreto independente da idade e os valores obtidos pela Equações 1, 2, 3, 6 e 7 está mostrado na Figura $6 a, 6 b$ e $6 c$. 
Como a NBR 6118 (ABNT, 2014) e a FIB (FEDERATION..., 2010) não especificam o valor para o dolomito, foi adotado o valor de 1,1, que é um valor citado pelo Ibracon (INSTITUTO..., 2003) para calcário sedimentar.

Todas as equações descrevem a tendência do comportamento no módulo de elasticidade tendo em vista a resistência do concreto, porém se percebe que as equações propostas pelas normas NBR 6118
(ABNT, 2007) e ACI 318 (AMERICAN..., 2011) apresentaram valores próximos, mas inferiores aos resultados experimentais. Dessa forma, a alteração realizada pela NBR 6118 (ABNT, 2014) foi justificada, pois permitiu que os valores calculados fossem representativos dos valores experimentais por tipo de agregado. Já a norma EUROCODE (2004) estimou todos os valores encontrados bem acima das demais equações e dos resultados experimentais.

Figura 5 - $E_{c i}$ em função do $f_{c}$ dos concretos estudados para todas as idades e as equações que melhor se ajustaram aos resultados encontrados para os concretos (a) BA-UDIA, (b) DO-PA e (c) BA-UBERA
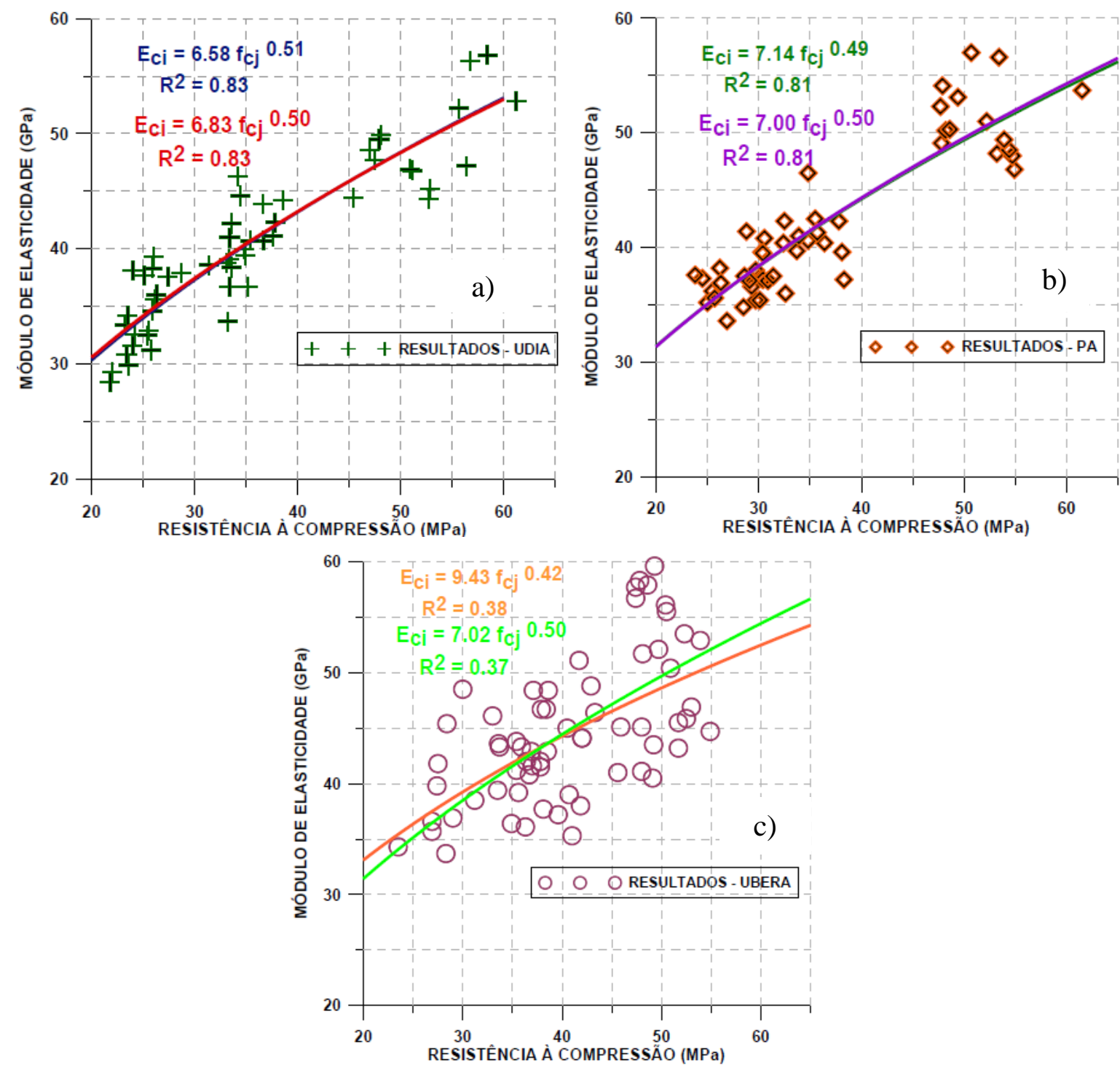
Figura 6 - Comparação dos resultados experimentais de $E_{c i}$ com os valores de $E_{c i}$ obtidos pelas equações propostas pelas normas e códigos para os concretos (a) BA-UDIA, (b) DO-PA e (c) BA-UBERA

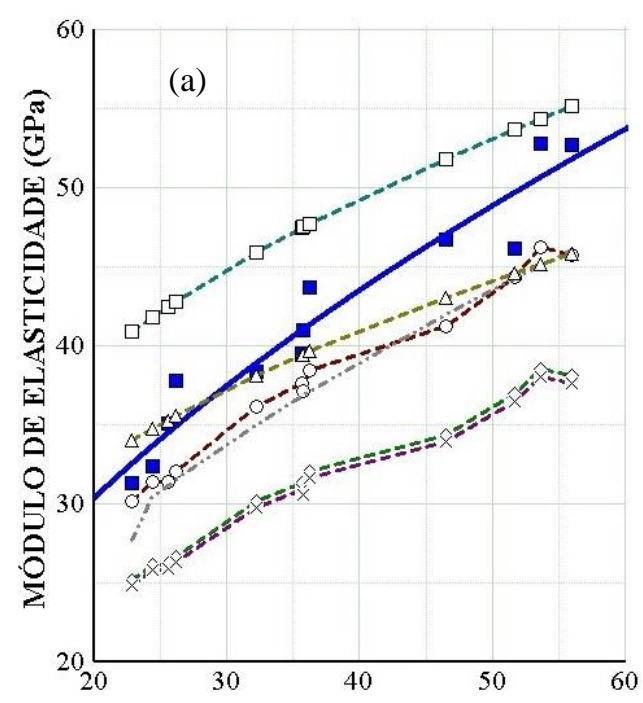

RESISTÊNCIA À COMPRESSÃO (MPa)

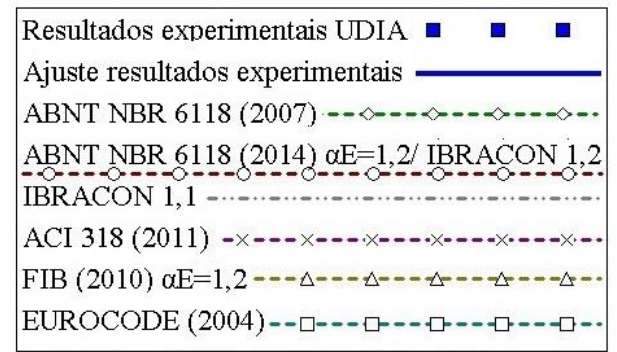

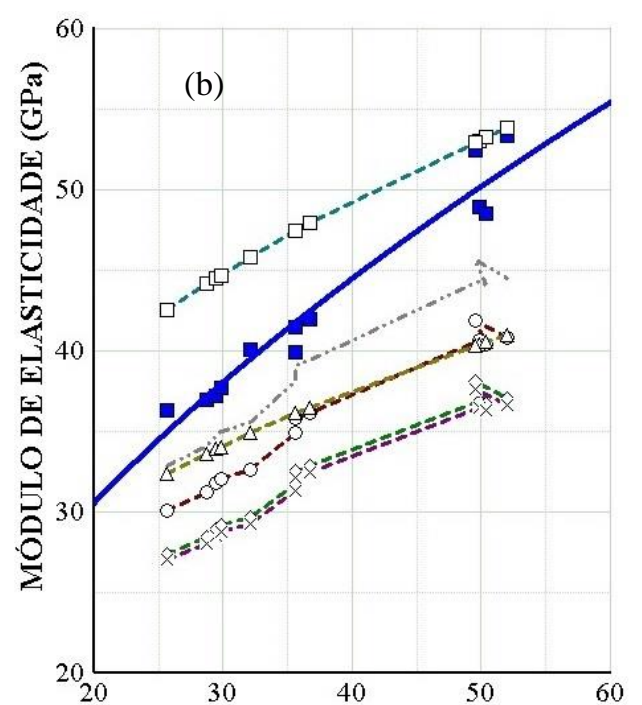

RESISTÊNCIA À COMPRESSÃO (MPa)

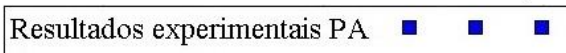

Ajuste resultados experimentais

ABNT NBR $6118(2007)--\diamond---\prec--\prec \diamond---\diamond-$

ABNT NBR $6118(2014) \alpha \mathrm{E}=1,1 / \mathrm{IBRACON} 1,1$

IBRACON 1,2

ACI 318 (2011)

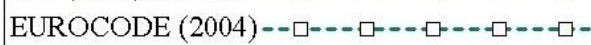

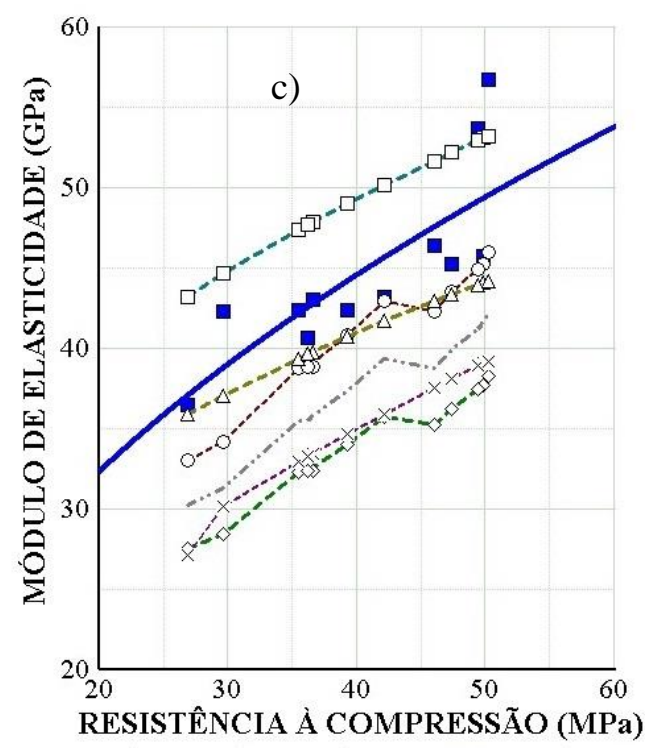

Resultados experimentais UBERA — a

Ajuste resultados experimentais

ABNT NBR 6118 (2007)

ABNT NBR $6118(2014) \alpha E=1,2 /$ IBRACON 1,2

IBRACON 1,1

ACI 318 (2011)

FIB (2010) $\alpha E=1,2-\cdots \Delta-\cdots \Delta---\Delta-\cdots-\Delta---\Delta-$

EUROCODE (2004) --口--- $\square---\square---\square+---\square-\cdot$ 
A equação proposta pelo FIB Model Code (FEDERATION..., 2010) foi o que mais se aproximou dos resultados encontrados. Todavia, para os concretos produzidos com BA, tal equação conduziu a valores superiores aos encontrados para os traços C20-BA-UDIA, C30-BA-UDIA, C20BA-UBERA, ao passo que para os concretos $\mathrm{C} 30$ BA-UBERA e da classe C40-BA os valores foram inferiores. A Equação 2 e a Equação 9 (Ibracon) permitiram a obtenção de valores que se ajustaram aos dados experimentais independentemente do agregado graúdo utilizado.

As equações propostas pelo FIB Model Code (FEDERATION..., 2010) e NBR 6118 (ABNT, 2014) foram as que mais se aproximaram dos resultados encontrados.

\section{Conclusões}

$\mathrm{Na}$ análise da influência do tipo de agregado graúdo no módulo de elasticidade de três classes de concreto C20, C30 e C40 foi possível verificar que o concreto produzido com agregado dolomítico, independentemente da classe de resistência, apresentou menor ganho de $\mathrm{f}_{\mathrm{c}}$ e de $\mathrm{E}_{\mathrm{ci}}$ dos 7 aos 56 dias. A porosidade do agregado controla a capacidade do agregado de restringir a deformação da matriz. Assim, o agregado DO de origem sedimentar, mais poroso, apresentou menor capacidade de restringir as deformações.

De forma geral, com exceção das idades de 14 dias e 28 dias do concreto produzido com agregado BAUBERA, os desvios-padrão das resistências foram superiores aos do módulo de elasticidade. Utilizando a função exponencial, foram obtidas as equações que melhor se ajustam aos resultados encontrados. O valor de R2 foi em torno de 0,8 . Entretanto, o concreto preparado com agregado graúdo procedente de Uberaba apresentou uma dispersão maior que os demais e o coeficiente de determinação do ajuste foi de 0,37 , ou seja, o modelo não representa o comportamento da amostra.

A análise de variância indicou que os agregados pouco influenciaram nos resultados dos módulos de elasticidade. Os dois basaltos utilizados tinham massas específicas com pequena diferença, daí supõe-se que os módulos de elasticidade das rochas também devem ser próximos, portanto a influencia no $E_{\mathrm{ci}}$ foi pequena. Quando analizados os concretos com os basaltos e com o dolomito, apesar da diferença entre as massas específicas ser de $6,7 \%$, não houve influência significativa no $\mathrm{E}_{\mathrm{ci}}$.

As equações propostas pelas normas NBR 6118 (ABNT, 2007) e ACI 318 (AMERICAN..., 2011) apresentaram valores de $\mathrm{E}_{\mathrm{ci}}$ próximos entre si e inferiores aos experimentais. Já a equação proposta pelo EUROCODE 2 (2004) estimou valores superiores aos resultados encontrados. A proposta de acrescentar fatores de correção em função do tipo de agregado se mostrou eficiente visto que a Equação 2, proposta pela NBR 6118 (ABNT, 2014), se aproximou mais dos resultados experimentais que a anteriormente proposta pela NBR 6118 (ABNT, 2007) que não considerava diferenças no tipo de agregado. As equações propostas pela FIB (FEDERATION..., 2010) e pela NBR 6118 (ABNT, 2014) com $\alpha E$ de 1,2 foram as que mais se aproximaram dos resultados experimentais encontrados.

A utilização de coeficientes utilizados nas equações das normas que representem os tipos litológicos de rochas nem sempre resultam em valores compatíveis com os reais, pois diferentes jazidas podem produzir diferentes materiais. Como sugestão, pode-se iniciar estudos para verificar esses coeficientes em função da massa específica do agregado, que neste trabalho mostrou coerência.

É importante salientar que os resultados experimentais devem, em geral, ser superiores aos calculados pelas normas que devem fornecer valores que preservem a segurança. Outro aspecto pertinente ao estudo realizado é a utilização de normas internacionais nas análises. Cada país desenvolve suas normas de forma a gerar arranjos que levem a obter os níveis de segurança requeridos; portanto, a utilização de uma norma fora do contexto do país pode levar a conclusões equivocadas.

\section{Referências}

\author{
ALHADAS, M. F. S. Estudo da Influência do \\ Agregado Graúdo de Diferentes Origens \\ Mineralógicas nas Propriedades Mecânicas do \\ Concreto. Belo Horizonte, 2008. Dissertação \\ (Mestrado em Engenharia Civil) - Escola de \\ Engneharia, Universidade Federal de Minas \\ Gerais, Belo Horizonte, 2008.
}

AMERICAN CONCRETE INSTITUTE. ACI 318M-11: structural building code. Michigan, 2011.

ASSOCIAÇÃO BRASILEIRA DE NORMAS TÉCNICAS. NM 67: concreto: determinação da consistência pelo abatimento do tronco de cone. Rio de Janeiro, 1998.

\section{ASSOCIAÇÃO BRASILEIRA DE NORMAS}

TÉCNICAS. NBR 8522: concreto: determinação do módulo estático de elasticidade à compressão. Rio de Janeiro, 2008. 
ASSOCIAÇÃO BRASILEIRA DE NORMAS TÉCNICAS. NBR 6118: projeto de estruturas de concreto: procedimento. Rio de Janeiro, 2007.

ASSOCIAÇÃO BRASILEIRA DE NORMAS TÉCNICAS. NBR 6118: projeto de estruturas de concreto: procedimento. Rio de Janeiro, 2014.

BARBOSA, I. L. S. Influência dos Agregados Graúdos da Região de Goiânia no Módulo de Deformação Tangente Inicial do Concreto. Goiânia, 2009. Dissertação (Mestrado em Engenharia Civil) - Escola de Engenharia, Universidade Federal de Goiás, Goiânia, 2009.

BESHR, H.; ALMUSALLAM, A. A.; MASLEHUDDIN, M. Effect of Coarse Aggregate Quality on the Mechanical Properties of High Strength Concrete. Constructions and Building Materials, v. 17, n. 1, p. 97-103, mar. 2003.

DIOGENES, H. J. F. et al. Determinação do Módulo de Elasticidade do Concreto a Partir da Resposta Acústica. Revista Ibracon de Estruturas e Materiais, São Paulo, v. 4, n.5, 2011.

EUROPEAN COMMITTEE FOR STANDARD:

EN 1992-1-1: Eurocode 2: design of concrete structures: part 1: general rules and rules for buildings. London, 2004.

FEDERATION INTERNATIONALE DU BETON. Fib Bulletin 55: Model Code 2010, First complete draft, v. 1, 2010.

INSTITUTO BRASILEIRO DO CONCRETO. Pratica Recomendada IBRACON-Comentários Técnicos NB-1. IBRACON, 2003.

HELENE, P. R. L.; ANDRADE, T. Concreto de Cimento Portland. In: ISAIA, G.C. (Org). Materiais de Construção Civil e Princípios de Ciência e Engenharia de Materiais. São Paulo: IBRACON, 2010.

MARRIAGA, J. L.; YÉPEZ, L. G. L. Effect of Sedimentary and Metamorphic Aggregate on the Static Modulus of Elasticity of High-Strength Concrete. Dyna, v. 78, n. 170, Medellin, dec. 2011.

MASSUCATO, C. J.; BASSANI, F.; PAULON, V. A. Contribuição Experimental Sobre o Módulo de Elasticidade Secante para Determinação de Esforços Solicitantes e Verificação de Estados Limites de Serviço. In: CONGRESSO BRASILEIRO DE CONCRETO, 45, Vitória, 2003. Anais... IBRACON, 2003.
MEHTA, P. M.; MONTEIRO, P. J. M. Concreto: microestrutura, propriedades e materiais. São Paulo: IBRACON, 2014.

MELO NETO, A. A.; HELENE, P. R. L. Módulo de Elasticidade: dosagem e avaliação de modelos de previsão do módulo de elasticidade de concretos. In: CONGRESSO BRASILEIRO DE CONCRETO, 44, Belo Horizonte, 2002. Anais... Belo Horizonte, 2002.

MONTIJA, F. C. Aspectos da Variabilidade Experimental do Ensaio de Módulo de Deformação do Concreto. São Paulo, 2007. Dissertação (Mestrado em Engenharia Civil) Escola de Engenharia, Universidade de São Paulo, São Paulo, 2007.

NEVILLE, A. M. Propriedades do Concreto. São Paulo: PINI, 1997.

NUNES, F. W. G. Resistência e Módulo de Elasticidade de Concretos Usados no Rio de Janeiro. Rio de Janeiro, 2005. Dissertação (Mestrado em Engenharia Civil) - Escola de Engenharia, Universidade Federal do Rio de Janeiro, Rio de Janeiro, 2005.

PACHECO, R. F. R. Análise do Módulo de Elasticidade e Resistência à Compressão de Concretos Produzidos em Centrais na Grande Vitória: experimentais e estatística. Vitória, 2006. Dissertação (Mestrado em Engenharia Civil) Escola de Engenharia, Universidade Federal do Espírito Santo, Vitória, ES, 2006.

PEDROSO, F. L. Módulo de Elasticidade é Parâmetro Fundamental Para a Durabilidade da Estrutura de Concreto. Revista Concreto e Construções, v. 48, 2007.

ZHOU, F. P.; LYDON, F. D.; BARR, B. I. G. Effect of Coarse Aggregate on Elastic Modulus and Compressive Strength of High Performance Concrete. Cement and Concrete Research, v. 25, p. 177-186, jan. 1995.

YANG, C. C. et al. Aggregate Effect on Elastic Moduli of Cement: based composite materials. Journal of Marine Science and Technology, v. 3, n. 1, pp. 5-10, 1995.

\section{Agradecimentos}

Os autores agradecem a ajuda financeira da Capes e da Fapemig. 


\section{Antonio Carlos dos Santos}

Faculdade de Engenharia Civil | Universidade Federal de Uberlândia | Av. João Naves de Avila, 2121, Bloco 1Y, Campus Santa Monica, Santa Monica | Uberlândia - MG - Brasil | CEP 38400-902 | Tel.: (34) 3239-4170 Ramal 225 | E-mail: acds@feciv.ufu.br

\section{Angela Maria de Arruda}

Universidade Federal de Uberlândia | E-mail: angelaarrudassp@gmail.com

\section{Turibio José da Silva}

Faculdade de Engenharia Civil | Universidade Federal de Uberlândia | E-mail: tjdsilva@gmail.com

Paula de Carvalho Palma Vitor

Universidade Federal de Uberlândia | E-mail: ppalmavitor@gmail.com

\section{Revista Ambiente Construído}

Associação Nacional de Tecnologia do Ambiente Construído

Av. Osvaldo Aranha, $99-3^{\circ}$ andar, Centro

Porto Alegre - RS - Brasil

CEP $90035-190$

Telefone: +55 (51) 3308-4084

Fax: +55 (51) 3308-4054

www.seer.ufrgs.br/ambienteconstruido

E-mail: ambienteconstruido@ufrgs.br 